\title{
Efficacy and safety of twice-daily rabeprazole maintenance therapy for patients with reflux esophagitis refractory to standard once-daily proton pump inhibitor: the Japan-based EXTEND study
}

\author{
Yoshikazu Kinoshita ${ }^{1} \cdot$ Mototsugu Kato $^{2} \cdot$ Mitsuhiro Fujishiro $^{3} \cdot$ Hironori Masuyama $^{4} \cdot$ \\ Ryo Nakata $^{5}$ - Hisanori Abe ${ }^{6}$ Shinji Kumagai ${ }^{7}$ Yasushi Fukushima ${ }^{8}$. \\ Yoshiumi Okubo $^{9}$ - Seiichiro Hojo ${ }^{10} \cdot$ Motoyasu Kusano $^{11}$
}

Received: 6 September 2017 / Accepted: 17 November 2017/Published online: 29 November 2017

(c) The Author(s) 2017. This article is an open access publication

\begin{abstract}
Background Rabeprazole at 10 or $20 \mathrm{mg}$ twice daily (b.i.d.) has been reported to be highly effective in the treatment of proton pump inhibitor (PPI)-resistant reflux esophagitis (RE) that is refractory to the standard oncedaily PPI regimen. We evaluated the efficacy and safety of rabeprazole maintenance therapy at $10 \mathrm{mg}$ once daily (q.d.) or b.i.d. for longer than 8 weeks.

Methods Patients with RE refractory to standard PPI regimens for at least 8 weeks were enrolled. They were treated with rabeprazole at 10 or $20 \mathrm{mg}$ b.i.d. for 8 weeks during the open-label treatment period. After endoscopic
\end{abstract}

Yoshikazu Kinoshita

kinosita@med.shimane-u.ac.jp

1 Department of Internal Medicine II, Faculty of Medicine, Shimane University, 89-1, Enya-cho, Izumo,

Shimane 693-8501, Japan

2 Department of Gastroenterology, National Hospital Organization Hakodate Hospital, Hakodate, Hokkaido, Japan

3 Department of Endoscopy and Endoscopic Surgery, Graduate School of Medicine, The University of Tokyo, Tokyo, Japan

4 Masuyama Gastrointestinal Clinic, Ohtawara, Tochigi, Japan

5 Japanese Red Cross Medical Center, Tokyo, Japan

6 Arita GI Hospital, Oita, Oita, Japan

7 Nakajima Hospital, Sendai, Miyagi, Japan

8 Tokyo-Eki Center-Building Clinic, Tokyo, Japan

9 Clinical Development Department, EA Pharma Co., Ltd, Tokyo, Japan

10 Clinical Data Science Department, Eisai Co., Ltd, Tokyo, Japan

11 Department of Gastroenterology, Gunma University Hospital, Maebashi, Gunma, Japan examination, those with confirmed healing entered the subsequent double-blind maintenance therapy. During this period, the subjects were randomized to receive rabeprazole $10 \mathrm{mg}$ q.d. (control) or $10 \mathrm{mg}$ b.i.d. The primary endpoint was the endoscopic no-recurrence rate at Week 52.

Results In total, 517 subjects entered the treatment, and 359 subjects continued on maintenance therapy. The full analysis set for central assessment included 343 subjects. The no-recurrence rate at Week 52 was significantly higher in the b.i.d. group $\left(73.9 \% ; p<0.001, \chi^{2}\right.$ test) than in the q.d. group $(44.8 \%)$. In particular, the b.i.d. regimen was more effective in all subgroups with Los Angeles Classification Grade B to D at treatment entry.

Conclusions In the maintenance treatment of PPI-resistant $\mathrm{RE}$, rabeprazole at $10 \mathrm{mg}$ b.i.d. exerted a stronger recurrence-preventing effect than $10 \mathrm{mg}$ q.d. over 52 weeks. No particular safety issues were noted during long-term administration.

ClinicalTrials.gov number: NCT02135107.

Keywords Endoscopy · GERD · Los Angeles

Classification · Proton pump inhibitor · Reflux esophagitis

\section{Introduction}

The numbers of patients suffering from reflux esophagitis (RE) have reportedly been increasing since the 1990s in Japan. The causes include a higher prevalence of risk factors for RE due to rising obesity rates [1], elevated gastric acid secretion associated with dietary and other lifestyle changes, and increased gastric acid secretion due to a lower prevalence of Helicobacter pylori-infected patients [2-4]. Proton pump inhibitors (PPI) have been 
proven to be highly effective in the treatment of RE, but the disease eventually recurs in $70-80 \%$ of patients [5]. Thus, maintenance therapy is important for prevention of recurrence and complications [6]. Maintenance therapy with PPI is regarded as highly effective [7] and as providing favorable cost-benefit performance. Specifically, rabeprazole taken once daily (q.d). has been shown to be effective as maintenance therapy for up to 5 years [8].

While PPI is widely used as the first-line therapy for RE, $6-15 \%$ of patients have PPI-refractory RE, such that a standard once-daily PPI regimen is ineffective for healing esophageal mucosal breaks or improving symptoms [9]. In these patients, lower quality of life (QOL) and work loss are reported [10, 11]. PPI-refractory RE might be attributable to nocturnal gastric acid breakthrough and the extensive metabolizer (EM) phenotype associated with the cytochrome P450 2C19 (CYP2C19) genotype. Standard PPI regimens are reportedly insufficient for suppressing nocturnal acid reflux in particular [12,13]. Patients with PPI-refractory RE are considered to have more frequent nocturnal reflux symptoms and sleep disorders due to longer retention of regurgitated gastric acid in the esophagus $[14,15]$.

Rabeprazole is reportedly more effective in suppressing nocturnal gastric acid secretion as a twice-daily (b.i.d.) regimen than as a q.d. regimen at the same daily dose [16]. In addition, the efficacies of PPI differ among individuals depending on genetic polymorphism in the hepatic drug metabolizing enzyme CYP2C19, although the impact of rabeprazole is considered to be less significant than that of other PPIs [17].

Based on reports that RE patients in the remission phase have elevated basal secretion similar to that in the active phase [18, 19], maintenance therapy for PPI-refractory RE requires continued potent inhibition of acid secretion after initial treatment.

The Japanese Society of Gastroenterology Clinical Practice Guidelines for Gastroesophageal Reflux Disease (GERD) recommend the rabeprazole b.i.d. regimen for treating PPI-refractory RE [16, 20], but evidence supporting efficacy is available only up to 8 weeks. To demonstrate the efficacy of the divided dosing regimen for longer than 8 weeks, this study was conducted to verify the efficacy and safety of rabeprazole $10 \mathrm{mg}$ b.i.d. as maintenance therapy for PPI-refractory RE.

\section{Methods}

\section{Study design and protocol}

This was a multicenter, randomized, parallel-group, double-blind comparative study evaluating the efficacy and safety of rabeprazole $10 \mathrm{mg}$ b.i.d. for 52 weeks as maintenance therapy. In total, 85 Japanese sites participated in this study.

This study was conducted in compliance with the ethical principles based on the Declaration of Helsinki-Ethical Principles, the Pharmaceuticals Affairs Law, and the standard operating procedures and study protocol established by the sponsor.

The study protocol, informed consent form, and their revisions were approved by the institutional review board and the director at each study site prior to study initiation. The study consisted of an 8-week treatment period followed by a 52 -week maintenance therapy period. During the treatment period, patients with PPI-refractory RE, that is refractory to the standard once-daily PPI regimen, were treated with rabeprazole 10 or $20 \mathrm{mg}$ b.i.d. in an open-label design. During the maintenance therapy period, subjects with endoscopically confirmed healing during the treatment period were randomized to receive rabeprazole $10 \mathrm{mg}$ q.d. or $10 \mathrm{mg}$ b.i.d. (Fig. 1).

The dose level during the treatment period was assigned according to the disease grading of the Los Angeles Classification [21, 22] based on endoscopic findings (physician's assessment) at treatment entry: $10 \mathrm{mg}$ b.i.d. for subjects with Grade A or B and $20 \mathrm{mg}$ b.i.d. for those with Grade C or D. Subject allocation during maintenance therapy was determined by a third-party organization (Bell Medical Solutions, Tokyo, Japan), using the endoscopic findings at treatment entry as factors for stratified allocation; the subjects were evenly randomized to the $10 \mathrm{mg}$ q.d. and $10 \mathrm{mg}$ b.i.d. groups. During the maintenance therapy period, we used placebo tablets which had exactly the same appearance as the rabeprazole $10 \mathrm{mg}$ tablets, the active drug. Subjects in the q.d. group took the active drug in the morning and placebo tablets in the evening, while subjects in the b.i.d group took the active drug both in the morning and in the evening. Subjects, investigators, and all other clinical study staff members were blinded to patient assignment during the maintenance therapy period.

Upper gastrointestinal endoscopy was performed using the Los Angeles Classification for eligibility assessment at treatment entry, evaluation of healing at Week 8 of treatment (before entering maintenance therapy), and evaluation of recurrence at Weeks 12, 24, and 52 of maintenance therapy. Subjects endoscopically confirmed to have unhealed disease at Week 8 of treatment were withdrawn, and those endoscopically confirmed to have recurrence during maintenance therapy completed the study upon confirmation.

Concomitant use of drugs possibly affecting the efficacy assessment, as well as contraindicated drugs, including PPIs, potassium-competitive acid blockers, $\mathrm{H}_{2}$ receptor antagonists, gastrointestinal prokinetic agents, protease 
Fig. 1 Study design. q.d. Once daily, b.i.d. twice daily
Treatment period ( 8 weeks) Maintenance therapy period (52 weeks)

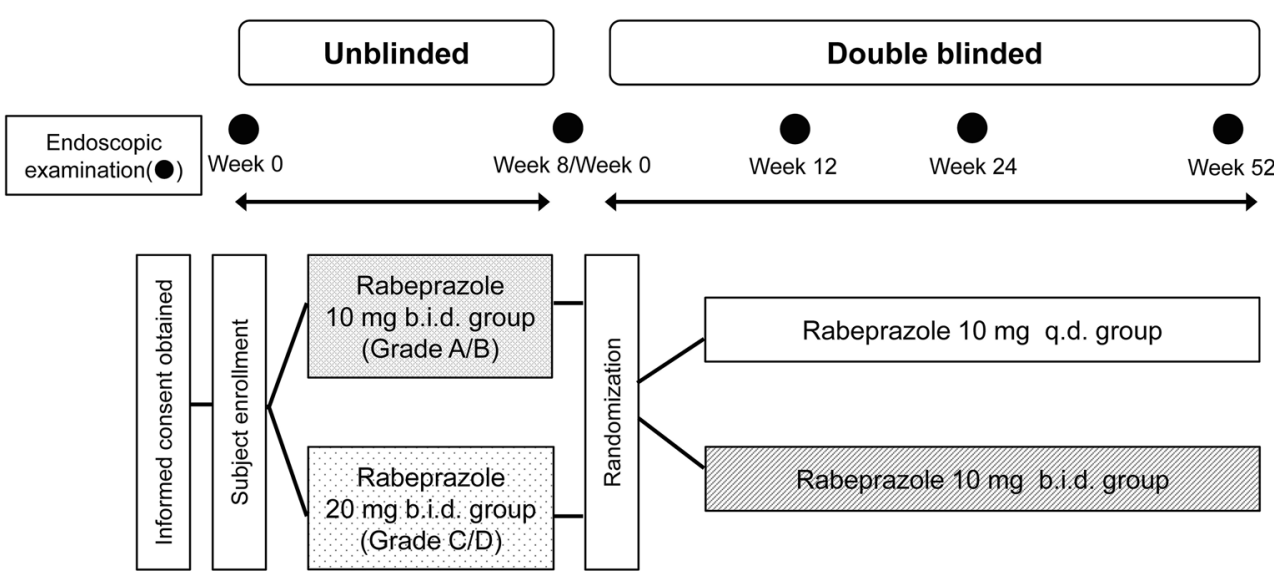

inhibitors, sodium alginate, atazanavir sulfate, and rilpivirine hydrochloride, were prohibited throughout the trial.

This trial was initiated in September 2013 and completed in May 2016.

\section{Subjects}

We enrolled patients with PPI-refractory RE, endoscopically confirmed to show no healing after at least 8 weeks of treatment with PPI at a standard q.d. dosing regimen approved in Japan, or even during maintenance therapy. Specifically, the standard doses were set at 10 or $20 \mathrm{mg} /$ day for rabeprazole (double dose was allowed), $30 \mathrm{mg} /$ day for lansoprazole, $20 \mathrm{mg} /$ day for omeprazole, and $20 \mathrm{mg} / \mathrm{day}$ for esomeprazole. At the time of performing endoscopic examination for study enrollment, patients were examined for hiatal hernia, according to the diagnostic criteria proposed by Makuuchi et al. [23], as well as for gastric polyps.

Patients were excluded if they had any of the following conditions: upper gastrointestinal tract bleeding within 8 weeks prior to study enrollment (including ongoing bleeding at enrollment); any serious disease, such as Barrett's esophagus $(\geq 3 \mathrm{~cm})$, Zollinger-Ellison syndrome, active gastric/duodenal ulcer; prior $H$. pylori eradication therapy within 6 months; existing or history of allergy to PPI; history of esophageal surgery or any other surgical intervention possibly affecting gastric acid secretion.

\section{Endpoints}

The primary endpoint was the no-recurrence rate based on endoscopic findings at Week 52 of maintenance therapy, as assessed by the Central Assessment Committee consisting of three endoscopists (MK, MK, and MF). These endoscopists performed their central assessments independently and then discussed the results if there were differences among them in the evaluation results.

The secondary endpoints were the no-recurrence rate based on physician-assessed endoscopic findings, the period from randomization to recurrence, and the time-course changes in the incidence and the resolution rate of heartburn (daytime, nighttime). The presence or absence of heartburn was assessed by the investigators during medical interviews. The heartburn incidence during each of the 7-day periods immediately before visiting the hospital was assessed on a scale of five stages based on the number of days with symptoms: 0 (no symptoms), $1-2,3-4,5-6$, and 7 (all) days. The incidence was tabulated by an analysis classifying the stages into two groups: "no symptom group" (0 days with symptoms) and "with symptoms group" (1 day or more with symptoms).

\section{Statistical analysis}

Assuming no-recurrence rates of 80 and $60 \%$ in the rabeprazole $10 \mathrm{mg}$ b.i.d. and q.d. dosing groups, respectively, a sample size of 218 patients (109 per group) was required to detect superiority of the b.i.d. regimen with a power of $90 \%$ and a two-sided significance level of $5 \%$ using the $\chi^{2}$ test. Thus, the target sample size was set at 300 for maintenance therapy, taking into consideration an approximately $25 \%$ withdrawal rate due to adverse events and other issues during the 52-week maintenance therapy period.

When determining the target sample size for the treatment, the healing rate of $75 \%$ was assumed to be appropriate for this period, based on the TWICE Study results [16]. Accordingly, a target sample size of 400 was set for the treatment to secure 300 patients for maintenance therapy. The protocol allowed enrollment of additional patients during the treatment period, if the number of patients 
eligible for enrollment in maintenance therapy failed to reach 300 .

Analysis was performed on the primary endpoint, the no-recurrence rate based on endoscopic findings at Week 52 of maintenance therapy, using the $\chi^{2}$ test, to demonstrate superiority of the $10 \mathrm{mg}$ b.i.d. regimen to the $10 \mathrm{mg}$ q.d. regimen with a two-sided significance level of 5\%. The analysis set included patients who completed maintenance therapy and were endoscopically confirmed to have recurrence during maintenance therapy and thereby withdrew from the study before Week 52. Furthermore, for the sensitivity analysis of the no-recurrence rate based on endoscopic findings at Week 52 of maintenance therapy, the Cochran Mantel-Haenszel (CMH) test was performed, using the disease grading according to the Los Angeles Classification (Grade A, B, C, or D) as a stratification factor, based on the endoscopic findings at treatment entry.

Similar analyses were performed on the physician's assessment, inter-group comparisons, and heartburn incidence. Furthermore, Kaplan-Meier curves were generated by plotting data from randomization to recurrence.

\section{Results}

\section{Characteristics and demographics of subjects}

Of the 896 patients giving consent, 517 met the eligibility criteria and began treatment, of whom 359 (69.4\%) completed the treatment (Fig. 2). The remaining 158 subjects $(30.6 \%)$ withdrew from treatment. The primary reason for withdrawal was a deviation from the inclusion criteria for the treatment (78 subjects) or being endoscopically confirmed as not having achieved healing at Week 8 of treatment (52 subjects). In a later review by the Central Assessment Committee, 78 subjects who had been considered ineligible for inclusion were judged to have Barrett's esophagus or (RE-related) mucosal break that had already been cured.

In total, 359 subjects began maintenance therapy, of whom $324(90.3 \%)$ completed the study. The remaining 35 subjects $(9.7 \%)$ withdrew from maintenance therapy, but 19 were included in the central assessment full analysis set (FAS). The primary reasons for withdrawal were adverse events (10 subjects), personal issues (9 subjects), and other (not having achieved healing at Week 8 of treatment in the central assessment, 9 subjects; discrepancy in evaluations between physicians and the Central Assessment Committee, 4 subjects; need for prohibited concomitant therapy, 1 subject; non-compliance with protocol, 1 subject; pregnancy, 1 subject). Thus, 343 subjects constituted the central assessment FAS, the demographics of which are summarized in Table 1, which shows that there were no significant differences between the q.d. and b.i.d. dosing groups.

\section{Efficacy}

The healing rate based on endoscopic findings at Week 8 was $87.4 \%$ (362/414 subjects) during the treatment period, for the central assessment FAS. The healing rate stratified by treatment regimen was $88.9 \%$ (304/342 subjects) for the $10 \mathrm{mg}$ b.i.d. and $80.6 \%$ (58/72 subjects) for the $20 \mathrm{mg}$ b.i.d. regimen.

The no-recurrence rate based on endoscopic findings at Week 52 of maintenance therapy, the primary endpoint, was $44.8 \%$ (73/163 subjects) in the $10 \mathrm{mg}$ q.d. group and $73.9 \%$ (119/161 subjects) in the $10 \mathrm{mg}$ b.i.d. group in the central assessment FAS; the no-recurrence rate was significantly higher in the $10 \mathrm{mg}$ b.i.d. group than in the $10 \mathrm{mg}$ q.d. group $\left(p<0.001, \chi^{2}\right.$ test). This significant difference emerged at Week 12 of maintenance therapy (Fig. 3).

The sensitivity analysis using the $\mathrm{CMH}$ test showed significantly higher values in the $10 \mathrm{mg}$ b.i.d. group than in the $10 \mathrm{mg}$ q.d. group, results which were consistent with those observed for the primary endpoint.

The no-recurrence rate based on physicians' assessments was significantly higher in the $10 \mathrm{mg}$ b.i.d. group $(75.9 \%, 120 / 158$ subjects) than in the $10 \mathrm{mg}$ q.d. group $\left(49.7 \%, 77 / 155\right.$ subjects) ( $p<0.001, \chi^{2}$ test). These rates were in line with the central assessment results.

The cumulative no-recurrence rate at Week 52 estimated by the Kaplan-Meier method was significantly higher in the $10 \mathrm{mg}$ b.i.d. group (71.4\%) than in the $10 \mathrm{mg}$ q.d. group $(41.5 \%)(p<0.001$, log-rank test; hazard ratio, $0.34)$.

In the subgroup analyses of the primary endpoint stratified by subject background characteristics, the time-course change by RE severity (Los Angeles Classification) at treatment entry revealed a higher preventive effect in the b.i.d. group (no-recurrence rate $\geq 75 \%$ in subjects with Grade $A / B, \geq 65 \%$ in those with Grade C) than in the q.d. group (Fig. 4).

Analyses of heartburn incidences during the daytime and the nighttime were conducted in subjects free of symptoms at maintenance therapy entry. The proportion of symptom-free subjects was maintained at the same level throughout the day (daytime-nighttime combined) and was significantly higher in the $10 \mathrm{mg}$ b.i.d. group than in the $10 \mathrm{mg}$ q.d. group, with symptom-free rates at Week 52 of $92.0 \%$ (126/137 subjects) and 76.8\% (106/138 subjects) $\left(p<0.001, \chi^{2}\right.$ test) (Fig. 5a). Analyses of the resolution rate of heartburn during the daytime and the nighttime were conducted in subjects who had symptoms at maintenance 
Fig. 2 Patient disposition based on central assessment. FAS Full analysis set

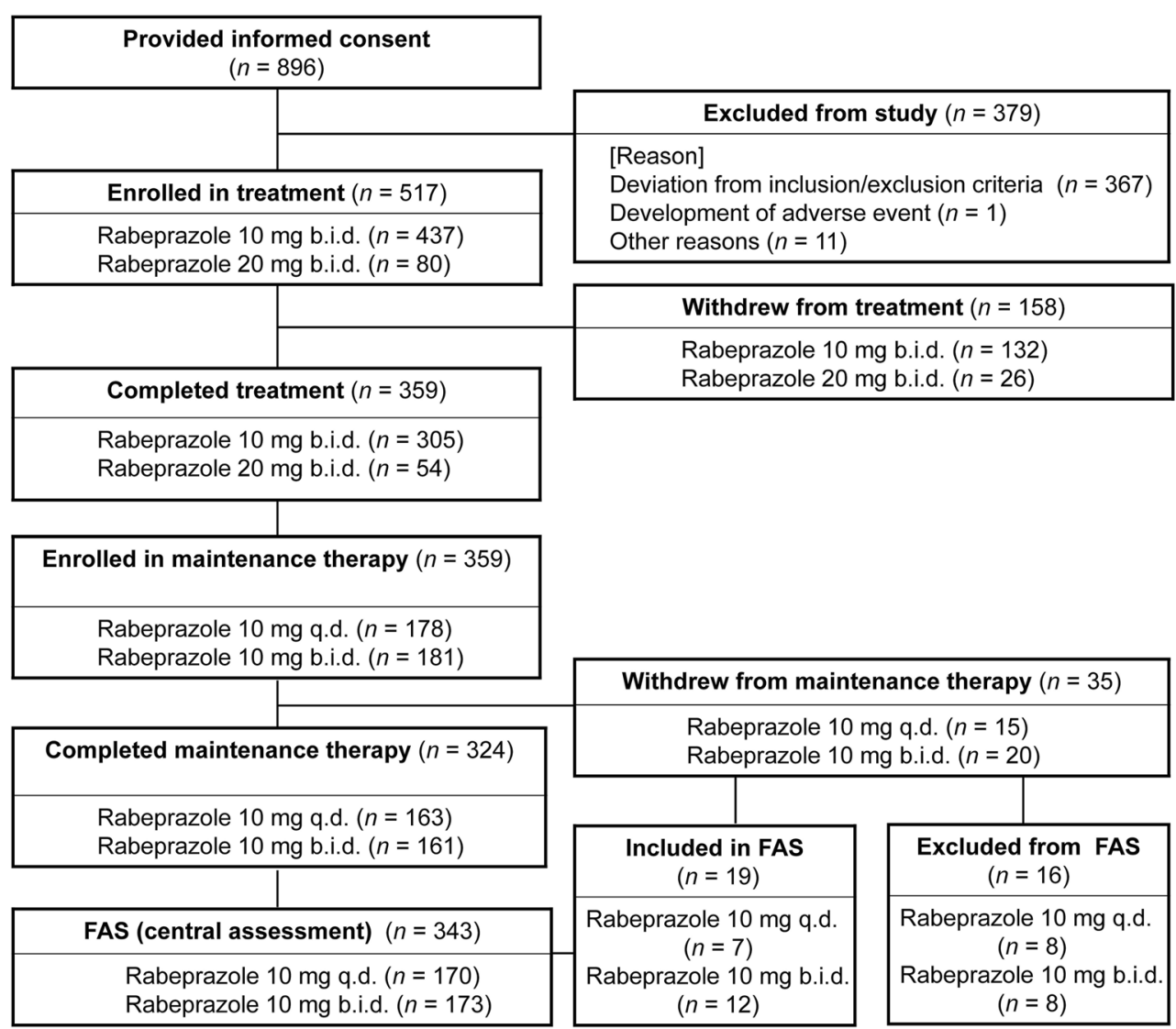

therapy entry. The resolution rate of heartburn throughout the day tended to be higher in the $10 \mathrm{mg}$ b.i.d. group $(62.5 \%, 15 / 24$ subjects) than in the $10 \mathrm{mg}$ q.d. group (54.2\%, 13/24 subjects) (Fig. 5b).

\section{Safety}

The incidence of adverse drug reactions was 3.9\% (7/178 subjects) for the $10 \mathrm{mg}$ q.d. group and $6.1 \%$ (11/181 subjects) for $10 \mathrm{mg}$ b.i.d. group. Adverse drug reactions with an incidence of at least $1 \%$ included stomatitis in the $10 \mathrm{mg}$ q.d. group, and diarrhea, elevated blood pressure, and increased blood thyroid-stimulating hormone in the $10 \mathrm{mg}$ b.i.d. group.

Serum gastrin levels (mean \pm standard deviation; upper limit of normal range $200 \mathrm{pg} / \mathrm{mL}$ ) at treatment entry were $297.4 \pm 302.16$ and $321.8 \pm 284.91 \mathrm{pg} / \mathrm{mL}$ in the $10 \mathrm{mg}$ b.i.d and $20 \mathrm{mg}$ b.i.d. groups, respectively. Serum gastrin levels at maintenance therapy entry were $359.1 \pm 256.63$ and $404.1 \pm 304.96 \mathrm{pg} / \mathrm{mL}$, and those at Week 52 of maintenance therapy were $283.3 \pm 228.23$ and $432.8 \pm 253.83 \mathrm{pg} / \mathrm{mL}$, in the $10 \mathrm{mg}$ q.d. and $10 \mathrm{mg}$ b.i.d. groups, respectively.

The cumulative gastric polyp incidence at Week 52 of maintenance therapy was $6.4 \%$ for the $10 \mathrm{mg}$ q.d. group and $13.3 \%$ for the $10 \mathrm{mg}$ b.i.d. group (Table 2). Gastric polyp types that developed by Week 52 in the $10 \mathrm{mg}$ q.d. group and the $10 \mathrm{mg}$ b.i.d. group included fundic gland polyps (5.8 and $8.7 \%$, respectively), gastric hyperplastic polyps (1.2 and $4.0 \%)$ and other types (0 and 1.2\%) (note: some subjects were included in multiple categories).

\section{Discussion}

In the treatment of $\mathrm{RE}$, prevention of acid regurgitation into the esophagus, healing of mucosal breaks, and maintenance of mucosal healing status constitute the most important strategy for resolving subjective symptoms, improving QOL [24], and preventing complications [25]. Severe RE that is refractory to a standard PPI regimen is associated with more frequent nocturnal reflux symptoms and sleep disorder due to longer retention of regurgitated acid in the esophagus [26]. Progression into more severe esophageal acid reflux is associated with an increased incidence of Barrett's esophagus, advancement to esophageal adenocarcinoma, and the complications of esophageal hemorrhage and stenosis [27, 28]. Because PPI therapy is a form of supportive care for acid regurgitation, PPI-refractory $\mathrm{RE}$ with severe acid regurgitation requires potent 
Table 1 Demographics and baseline characteristics of subjects during the maintenance therapy period (central assessment full analysis set)

\begin{tabular}{|c|c|c|c|c|}
\hline Parameter & 10 mg q.d. $(n=170)$ & 10 mg b.i.d. $(n=173)$ & Total $(n=343)$ & $p$ value \\
\hline \multicolumn{4}{|l|}{ Age (years) ${ }^{\mathrm{b}}$} & 0.6612 \\
\hline$<65$ & $71(41.8)$ & $68(39.3)$ & $139(40.5)$ & \\
\hline$\geq 65$ & $99(58.2)$ & $105(60.7)$ & $204(59.5)$ & \\
\hline \multicolumn{4}{|l|}{ Sex } & 0.3748 \\
\hline Male & $102(60.0)$ & $112(64.7)$ & $214(62.4)$ & \\
\hline Female & $68(40.0)$ & $61(35.3)$ & $129(37.6)$ & \\
\hline \multicolumn{4}{|l|}{ Body mass index $\left(\mathrm{kg} / \mathrm{m}^{2}\right)$} & 0.6630 \\
\hline$<25.0$ & $99(58.2)$ & $96(55.5)$ & $195(56.9)$ & \\
\hline$\geq 25.0$ & $71(41.8)$ & $77(44.5)$ & $148(43.1)$ & \\
\hline \multicolumn{4}{|l|}{ History of RE prior to treatment entry } & 0.3005 \\
\hline No healing after $\geq 8$ weeks of PPI q.d. & $147(86.5)$ & $142(82.1)$ & $289(84.3)$ & \\
\hline Recurred during PPI maintenance therapy & $23(13.5)$ & $31(17.9)$ & $54(15.7)$ & \\
\hline \multicolumn{4}{|c|}{ Los Angeles Classification of reflux esophagitis at treatment entry } & 0.7765 \\
\hline Grade A & $84(49.4)$ & $81(46.8)$ & $165(48.1)$ & \\
\hline Grade B & $57(33.5)$ & $56(32.4)$ & $113(32.9)$ & \\
\hline Grade C & $27(15.9)$ & $32(18.5)$ & $59(17.2)$ & \\
\hline Grade D & $2(1.2)$ & $4(2.3)$ & $6(1.7)$ & \\
\hline \multicolumn{4}{|l|}{ Spinal deformity } & 0.3714 \\
\hline Yes & $23(13.5)$ & $30(17.3)$ & $53(15.5)$ & \\
\hline No & $147(86.5)$ & $143(82.7)$ & $290(84.5)$ & \\
\hline \multicolumn{4}{|l|}{ Esophageal hiatal hernia classification } & 0.2817 \\
\hline $\mathrm{O}$ (absent) & $37(21.8)$ & $29(16.8)$ & $66(19.2)$ & \\
\hline $\mathrm{C}$ & $28(16.5)$ & $22(12.7)$ & $50(14.6)$ & \\
\hline $\mathrm{B}$ & $76(44.7)$ & $95(54.9)$ & $171(49.9)$ & \\
\hline A & $29(17.1)$ & $27(15.6)$ & $56(16.3)$ & \\
\hline \multicolumn{5}{|l|}{ Heartburn at maintenance therapy entry } \\
\hline \multicolumn{4}{|l|}{ Daytime } & 1.0000 \\
\hline Yes & $20(11.8)$ & $21(12.1)$ & $41(12.0)$ & \\
\hline No & $150(88.2)$ & $152(87.9)$ & $302(88.0)$ & \\
\hline \multicolumn{4}{|l|}{ Nighttime } & 0.1920 \\
\hline Yes & $14(8.2)$ & $8(4.6)$ & $22(6.4)$ & \\
\hline No & $156(91.8)$ & $165(95.4)$ & $321(93.6)$ & \\
\hline \multicolumn{4}{|l|}{ Daytime or nighttime } & 0.7607 \\
\hline Yes & $26(15.3)$ & $24(13.9)$ & $50(14.6)$ & \\
\hline No & $144(84.7)$ & $149(86.1)$ & $293(85.4)$ & \\
\hline \multicolumn{4}{|c|}{ Sleep disorder due to nocturnal heartburn or acid regurgitation at maintenance therapy entry } & 0.4995 \\
\hline Yes & $5(2.9)$ & $3(1.7)$ & $8(2.3)$ & \\
\hline No & $165(97.1)$ & $170(98.3)$ & $335(97.7)$ & \\
\hline \multicolumn{4}{|c|}{ Serum gastrin level at maintenance therapy entry $(\mathrm{pg} / \mathrm{mL})$} & 0.2036 \\
\hline$<200$ & $51(30.0)$ & $39(22.5)$ & $90(26.2)$ & \\
\hline$\geq 200$ to $<400$ & $58(34.1)$ & $58(33.5)$ & $116(33.8)$ & \\
\hline$\geq 400$ & $61(35.9)$ & $76(43.9)$ & 137 (39.9) & \\
\hline \multicolumn{4}{|l|}{ CYP2C19 genotype ${ }^{c}$} & 0.2941 \\
\hline Homozygous EM & $78(45.9)$ & $72(41.6)$ & $150(43.7)$ & \\
\hline Heterozygous EM & $79(46.5)$ & $79(45.7)$ & $158(46.1)$ & \\
\hline $\mathrm{PM}$ & $13(7.6)$ & $22(12.7)$ & $35(10.2)$ & \\
\hline
\end{tabular}


Table 1 continued

\begin{tabular}{lccc}
\hline Parameter & $10 \mathrm{mg}$ q.d. $(n=170)$ & $10 \mathrm{mg}$ b.i.d. $(n=173)$ & Total $_{(n=343)}$ \\
\hline Anti-Helicobacter pylori immunoglobulin G antibody ${ }^{\mathrm{d}}$ & & 0.3685 \\
$\quad$ Positive & $8(4.7)$ & $13(7.5)$ & $21(6.1)$ \\
$\quad$ Negative & $162(95.3)$ & $160(92.5)$ & $322(93.9)$ \\
\hline
\end{tabular}

Categorical variable data are presented as numbers of subjects. Values in parentheses are the proportion (\%) of subjects in each dosing group relative to the number of subjects in the analysis set. The calculation excluded subjects with missing data

PPI proton pump inhibitor, q.d. once daily, $C Y P$ cytochrome P450, EM extensive metabolizer, $P M$ poor metabolizer

${ }^{a}$ Fisher's exact test was used

${ }^{\mathrm{b}}$ Age at consent acquisition

${ }^{\mathrm{c} C Y P} 2 \mathrm{C} 19$ genotype: $* 1 / * 1$ was homozygous EM, $* 1 / * 2$ and $* 1 / * 3$ were heterozygous EM, $* 2 / * 2, * 3 / * 3$, and $* 2 / * 3$ were PM

${ }^{\mathrm{d}}$ Titer: $\geq 10 \mathrm{U} / \mathrm{mL}$ was defined as positive, and $<10 \mathrm{U} / \mathrm{mL}$, as negative

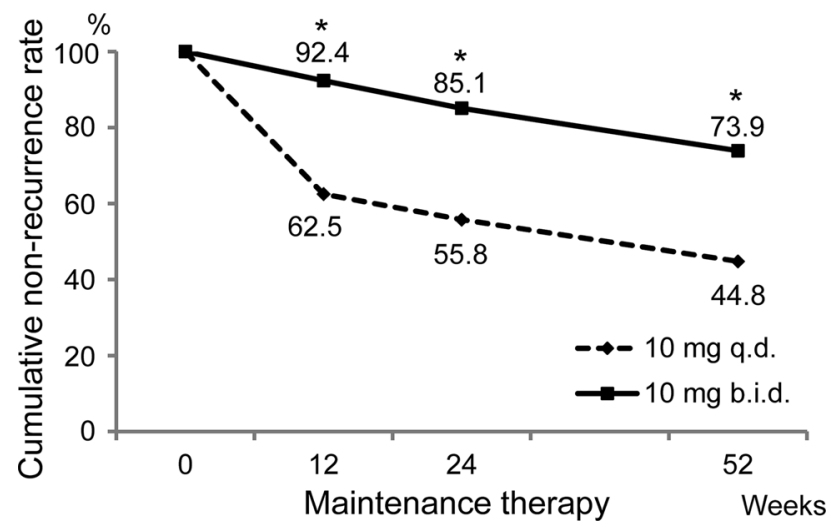

Fig. 3 Primary endpoint: no-recurrence rate based on endoscopic findings at Week 52 of maintenance therapy per central assessment. The no-recurrence rate was analyzed using the $\chi^{2}$ test, and the superiority of the rabeprazole $10 \mathrm{mg}$ b.i.d. regimen to the $10 \mathrm{mg}$ q.d. regimen was verified

inhibition of acid secretion by means of ongoing maintenance therapy.

This study registered patients with PPI-refractory RE who were assessed by physicians as not having achieved a cure after at least 8 weeks of treatment with a standard PPI regimen. However, the Central Assessment Committee later reviewed the subjects' eligibilities, and disqualified 78 judged to have Barrett's esophagus, or RE lesions that had already been cured, thus making them ineligible for this study. In the protocol of this study, short-distance, whole circumference endoscopic images of the gastroesophageal junction were to be submitted to the Central Assessment Committee, and an average of 3.3 endoscopic images per patient were submitted to the Central Assessment Committee during each assessment period. If there were differences in the evaluation results among the three endoscopists in the Central Assessment Committee, a final agreement was reached after discussions among the three endoscopists.
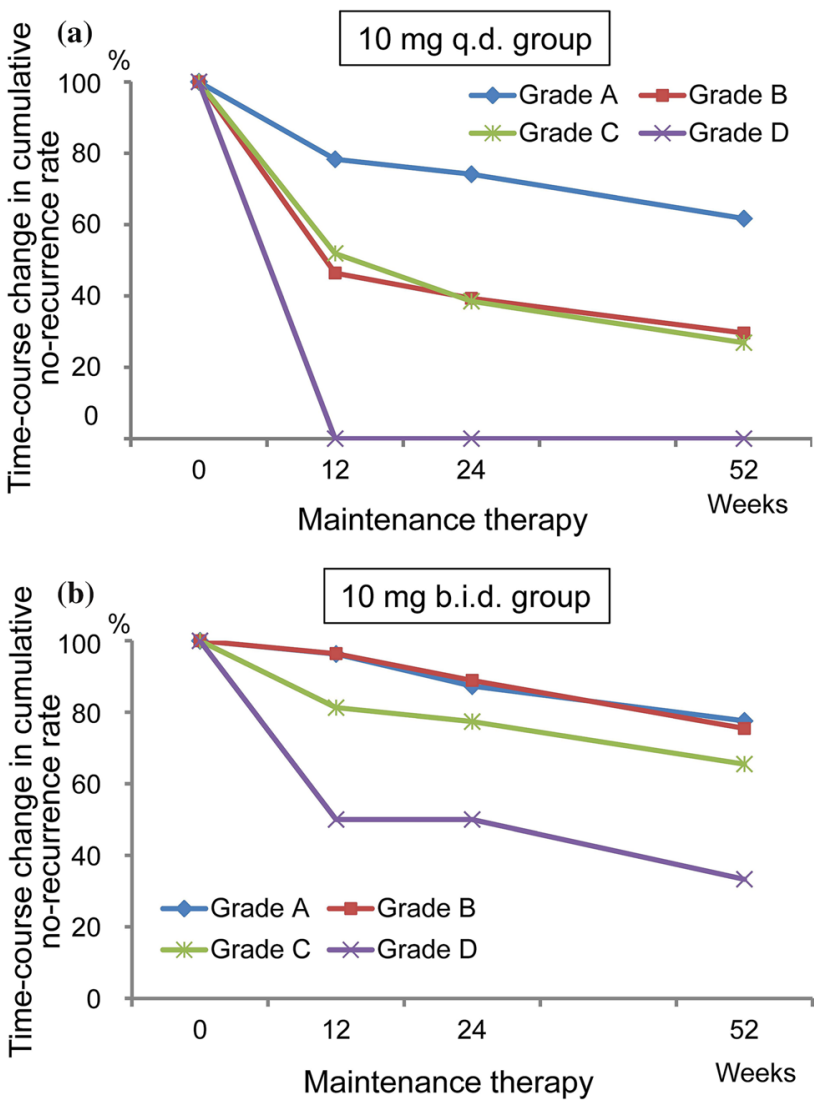

Fig. 4 Stratified analysis: time-course change in no-recurrence rate at Week 52 of maintenance therapy stratified by the Los Angeles Classification at treatment entry. a $10 \mathrm{mg}$ q.d. group, b $10 \mathrm{mg}$ b.i.d. group

The no-recurrence rate, based on endoscopic findings at Week 52 of maintenance therapy per central endoscopy assessment, was significantly higher in the $10 \mathrm{mg}$ b.i.d. group than in the $10 \mathrm{mg}$ q.d. group. The $\mathrm{CMH}$ test, which was carried out from the sensitivity analysis viewpoint with adjustment by the Los Angeles Classification based on endoscopic findings at treatment entry, revealed no 


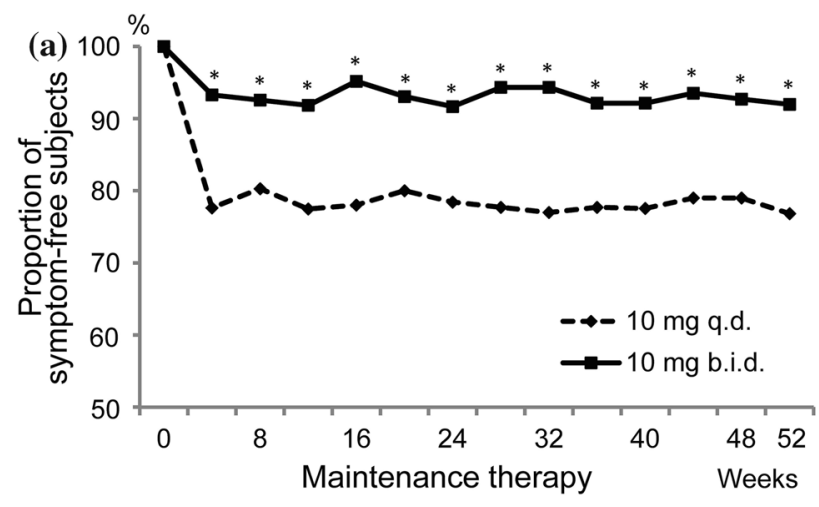

(b)

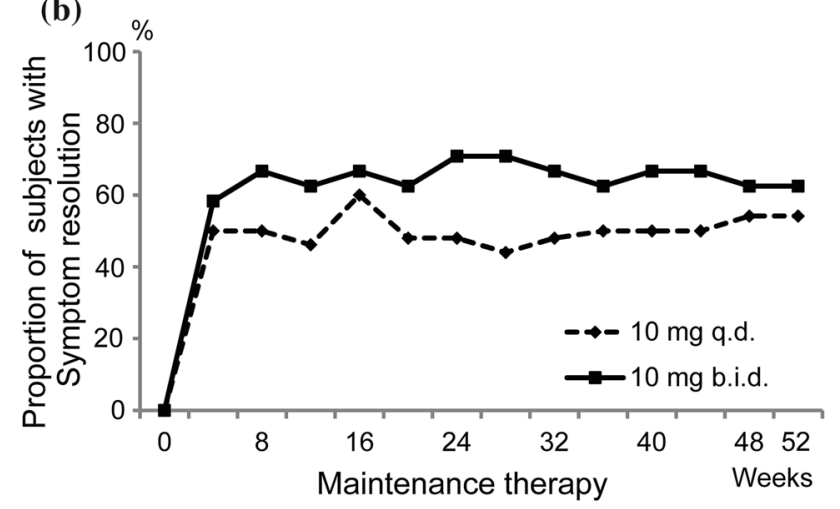

Fig. 5 Secondary endpoint: time-course changes in suppression and occurrence rates of symptoms. a Proportion of symptom-free subjects (daytime/nighttime combined) among those without heartburn at maintenance therapy entry. b Proportion of subjects experiencing symptom resolution (daytime/nighttime combined) among those with heartburn at maintenance therapy entry

difference from the primary results, i.e. the $10 \mathrm{mg}$ b.i.d. group showed significant superiority to the $10 \mathrm{mg}$ q.d. group. A previous study conducted by Shimatani et al. in $H$. pylori-negative healthy adults demonstrated intragastric $\mathrm{pH}>4$ holding time ratios of 49,59 and $71 \%$ in homo EM, hetero EM, and poor metabolizer (PM) subgroups, respectively, with a rabeprazole $10 \mathrm{mg}$ q.d. regimen, as well as ratios of 85,86 , and $99 \%$, respectively, with a rabeprazole $10 \mathrm{mg}$ b.i.d. regimen [29]. In the current trial, the inhibitory effects on acid secretion also correlated with the mucosal healing effect during maintenance therapy. Similar tendencies were indicated in the current trial between the central assessment and physicians' assessments in the endoscopic recurrence evaluation during maintenance therapy. Although evaluator-associated variability was reported for endoscopic diagnosis [30], such variation was limited in the current trial which employed sample image collection to achieve consistent assessment. In the protocol of this study, we had stipulated that the dosing timing of rabeprazole was to be "after breakfast" and "after dinner". Thus, we are confident that we were able to compare the q.d. and b.i.d. regimens in an appropriate manner. It has also been shown that acid secretion inhibition by rabeprazole is not affected by meals [31]. We therefore believe that a proper treatment effect was exerted.

In our subgroup analyses, stratification by RE severity at treatment entry revealed a recurrence rate exceeding $70 \%$ in Grades B to D subjects in the $10 \mathrm{mg}$ q.d. group. These observations support recommending a $10 \mathrm{mg}$ b.i.d. regimen as maintenance therapy for patients with PPI-refractory RE of Grade B or higher. Similarly, the no-recurrence rate was higher in the $10 \mathrm{mg}$ b.i.d. group than in the $10 \mathrm{mg}$ q.d. group of patients with PPI-refractory RE with severe

Table 2 Cumulative gastric polyp incidence at Week 52 of maintenance therapy (safety analysis set)

\begin{tabular}{|c|c|c|c|}
\hline \multirow[t]{2}{*}{ Category } & \multicolumn{2}{|l|}{ Therapy } & \multirow[t]{2}{*}{$p$ value $^{\mathrm{a}}$} \\
\hline & 10 mg q.d. $(n=178)$ & 10 mg b.i.d. $(n=181)$ & \\
\hline Gastric polyp present ${ }^{\mathrm{b}}(\%)$ & $11(6.4)$ & $23(13.3)$ & 0.0457 \\
\hline New gastric polyp developed & $11(6.4)$ & $19(11.0)$ & 0.1805 \\
\hline Existing gastric polyp increased/enlarged & $1(0.6)$ & $7(4.0)$ & 0.0672 \\
\hline Gastric polyp present during the treatment period ${ }^{\mathrm{b}}$ & $1(0.6)$ & $9(5.2)$ & - \\
\hline New gastric polyp developed & $1(0.6)$ & $5(2.9)$ & - \\
\hline Existing gastric polyp increased/enlarged & $0(0.0)$ & $6(3.5)$ & - \\
\hline Gastric polyp absent at maintenance therapy entry ${ }^{\mathrm{b}}$ & $10(5.8)$ & $14(8.1)$ & - \\
\hline New gastric polyp developed & $10(5.8)$ & $14(8.1)$ & - \\
\hline Existing gastric polyp increased/enlarged & $1(0.6)$ & $1(0.6)$ & - \\
\hline Gastric polyp absent (\%) & $160(93.6)$ & $150(86.7)$ & - \\
\hline
\end{tabular}

Subjects with gastric polyp data available for assessment in the safety analysis set were included in the analysis. Values are presented as the number of subjects, with the proportion (\%) of subjects in each dosing group given in parenthesis

${ }^{a}$ Fisher's exact test was used

${ }^{\mathrm{b}}$ Some subjects were included in multiple gastric polyp categories 
esophageal hiatal hernia which is known to be a cause of PPI-refractory RE [32].

In the evaluation of subjective symptoms, the proportion of symptom-free subjects (daytime-nighttime combined) was also higher in the b.i.d. than in the q.d. group, showing the superiority of the b.i.d. regimen to the q.d. regimen for the prevention of symptom onset. The symptom-free rate for heartburn changed until Week 8 of the maintenance therapy period, which might have been attributable to dose reduction from the treatment period to the maintenance therapy period. However, after Week 8 , the symptom-free rate remained essentially stable until the end of the maintenance therapy period. In particular, we observed that the heartburn symptoms appeared to be more likely to have developed in the groups in which the dose was changed from $20 \mathrm{mg}$ b.i.d.to $10 \mathrm{mg}$ b.i.d., from $20 \mathrm{mg}$ b.i.d. to $10 \mathrm{mg}$ q.d., or from $10 \mathrm{mg}$ b.i.d. to $10 \mathrm{mg}$ q.d.

The incidences of adverse drug reactions did not differ markedly between the two groups during the maintenance therapy period. Furthermore, long-term treatment with the b.i.d. regimen did not increase incidences of any specific events or the development of new events: the incidences were lower than those in a previous study evaluating rabeprazole $10 \mathrm{mg}$ or $20 \mathrm{mg}$ q.d. maintenance therapy for RE patients [8].

The target population of this study was PPI-refractory RE patients previously treated with PPIs for at least 8 weeks, and the subjects therefore had higher serum gastrin levels at the start of treatment. Following treatment initiation, serum gastrin levels increased slightly, but the mean serum gastrin levels remained stable without further increase during the maintenance therapy period. These results are consistent with those of a previous study [16], and the values were lower than in patients treated with potassium-competitive acid blockers [33].

The cumulative gastric polyp incidence was higher in the $10 \mathrm{mg}$ b.i.d. group (13.3\%) than in the $10 \mathrm{mg}$ q.d. group $(6.4 \%)$. On the other hand, the cumulative gastric polyp incidence at Week 52 estimated by the KaplanMeier method in the $10 \mathrm{mg}$ b.i.d. group was $15.7 \%$, slightly higher than the $12.7 \%$ in the $10 \mathrm{mg}$ q.d. group. These incidences were not markedly higher than those reported in a prospective study evaluating a $10 \mathrm{mg}$ q.d. maintenance therapy regimen for RE patients [34].

This study has three limitations: first, we did not evaluate the rabeprazole $20 \mathrm{mg}$ b.i.d. regimen as maintenance therapy. Because approximately $26 \%$ of subjects experienced recurrence even during 52-week maintenance therapy with the rabeprazole $10 \mathrm{mg}$ b.i.d. regimen, subjects with severe mucosal breaks (Los Angeles Classification Grades $\mathrm{C}$ and $\mathrm{D}$ ) after being given a standard PPI regimen, and then received treatment with the $20 \mathrm{mg}$ b.i.d. regimen, might have required the same dosing regimen during the maintenance therapy period. Second, the incidences of adverse events in the q.d. group might have been underestimated: the protocol specified study withdrawal upon endoscopic recurrence, which might have resulted in a shorter mean exposure to the study drug in the q.d. group which had a higher recurrence rate than the b.i.d. group. Third, the study period was 52 weeks such that no data are available on efficacy and safety beyond 52 weeks. Future investigation is warranted to evaluate the results of longer treatment.

In summary, this study demonstrated maintenance therapy with rabeprazole $10 \mathrm{mg}$ b.i.d. up to Week 52 to be significantly superior to rabeprazole $10 \mathrm{mg}$ q.d. in preventing RE recurrence in patients with PPI-refractory RE.

Acknowledgements We thank all patients and investigators participated in this study. The principal investigators at the following 85 study sites in Japan participated in this study: Sei Kurokawa and Takeshi Hagiwara from Sapporo-Kosei General Hospital, Sapporo; Izumi Tsunematsu from Touei Hospital, Sapporo; Hisato Hara from Medical Corporation Hakushoukai Sano Hospital, Asahikawa; Mutsubu Sugawara from Medical Corporation Jiseikai Higashiasahikawa Hospital, Asahikawa; Gakuyo Karasawa from Karasawa hospital, Asahikawa; Takayuki Goda from Ishikari Hospital Social Medical Corporation Pieta Society, Ishikari; Tomoyuki Ota from Sapporo Higashi Tokushukai Hospital, Sapporo; Hirohito Muramatsu Sapporo from Kiyota Hospital, Sapporo; Toshiharu Chikama from Chikama Gastro-Intestinal \& Internal Medicine Clinic, Sapporo; Ikuo Mitani from Mitani Gastrointestinal Clinic, Sapporo; Masao Yamauchi from Yamauchi Internal Medicine Clinic, Sapporo; Shinji Kumagai from Nakajima Hospital, Sendai; Hiro-o Yamano from Akita Red Cross Hospital, Akita; Harufumi Oizumi from OIZUMI medical Clinic, Yamagata; Kazuhiko Aikawa from Igarashi Internal Medicine Surgery Clinic, Koriyama; Noriyuki Nishino from Southern TOHOKU Research Institute for Neuroscience Southern TOHOKU Medical Clinic, Koriyama; Masaaki Kanenobu from Yuai Memorial Hospital, Koga; Yoshinori Sakai from Tsuchiura Kyodo General Hospital, Tsuchiura; Hironori Masuyama from Masuyama Gastrointestinal Clinic, Ohtawara; Satoshi Ono from Sutoh Hospital, Annaka; Shigeyasu Kamata from Kamata iin, Saitama; Ko Nishikawa from Ageo Central General Hospital, Ageo; Keita Sasajima from Japanese Red Cross Saitama Hospital, Saitama; Yasuo Hosoda from Saitama National Hospital, Wako; Kazuhiro Sugitani from Japanese Red Cross Ogawa Hospital, Hiki; Youji Harada from Todachuo General Hospital, Toda; Shunji Fujimori from Nippon Medical School Chiba Hokuso Hospital, Inzai; Joji Yamamoto from Kamagaya General Hospital, Kamagaya; Rika Nakano and Hisato Maekawa from Japan Community Healthcare Organization Tokyo Takanawa Hospital, Tokyo; Katsuhiko Iwakiri, Noriyuki Kawami and Mariko Umezawa from Nippon Medical School Graduate school of Medicine, Tokyo; Katsutoshi Yoshida from Jiai Hospital, Tokyo; Shigeru Fujisaki from Fujisaki Hospital, Tokyo; Nobuyuki Matsuhashi from NTT Medical Center Tokyo, Tokyo; Ryo Nakata from Japanese Red Cross Medical Center, Tokyo; Tetsuya Tsurumachi from Tsurumachi Clinic, Tokyo; Naoto Kurihara from Tokyo Healthcare Foundation Nerima General Hospital, Tokyo; Toshiaki Terada from Terada Hospital, Medical Corporation Shunwakai, Tokyo; Hikaru Ishii from Shin-nihonbashi Ishii Clinic, Tokyo; Tomofumi Murakami from Shimokitazawa Tomo Clinic, Tokyo; Masae Banno from Banno Medical Clinic, Tokyo; Eiichi Sato from Sato Gastroenterological Medical Clinic, Tokyo; Yasushi Fukushima from Tokyo-Eki Center-Building Clinic, Tokyo; Akiko Sasaki from Shonankamakura General Hospital, Kamakura; 
Hiroaki Makiyama, Masaru Tauchi and Shinichiro Fukuda from Yokohama Shin-midori General Hospital, Yokohama; Tatsuji Komatsu from National Hospital Organization Yokohama Medical Center, Yokohama; Seiji Otsuka from Koukan Clinic, Kawasaki; Kenji Mukawa from Suwa Red Cross Hospital, Suwa; Takahisa Furuta from Hamamatsu University School of Medicine, Hamamatsu; Yasuhiko Maruyama from Fujieda Municipal General Hospital, Fujieda; Yasuhisa Yokoyama and Tadashi Yokoyama from Yokoyama Memorial Hospital, Nagoya; Naoto Kanemaki and Koji Nonogaki from Daido Hospital, Nagoya; Takashi Ando from Kyoto Kuramaguchi Medical Center, Kyoto; Norimasa Yoshida from Japanese Red Cross Kyoto Daiichi Hospital, Kyoto; Norihiko Watanabe from Hirakata Kohsai Hospital, Hirakata; Eiji Umegaki and Toshihisa Takeuchi from Osaka Medical College Hospital, Takatsuki; Yasuhiro Fujiwara from Osaka City University Graduate School of Medicine, Osaka; Kiyoshi Ashida and Hiroshi Yamashita from Saiseikai Nakatsu Hospital, Osaka; Souken Sai from Sai Gastroenterology Proctology Clinic, Fujiidera; Wataru Ono from Kishiwada Tokushukai Hospital, Kishiwada; Hiroshi Morikawa from Morikawa Clinic, Hirakata; Hiroyuki Ogawa from Nishinomiya Municipal Central Hospital, Nishinomiya; Naohisa Hashimoto from Yotsuba Clinic, Amagasaki; Tetsuro Inokuma from Kobe City Medical Center General Hospital, Kobe; Nobuo Aoyama from Aoyama Clinic, Kobe; Norihisa Ishimura from Shimane University Faculty of Medicine, Izumo; Hirofumi Fujishiro from Shimane Prefectural Central Hospital, Izumo; Toru Kawamura from Kawamura Internal Medical Clinic, Hiroshima; Takayuki Imada from Nippon Kokan Fukuyama Hospital, Fukuyama; Ikuta Tanaka from Takamatsu Municipal Hospital, Takamatsu; Tomoki Inaba from Kagawa Prefectural Central Hospital, Takamatsu; Mitsushige Shibatoge from Takamatsu Red Cross Hospital, Takamatsu; Tomoyuki Ninomiya from Ehime Prefectural Central Hospital, Matsuyama; Shuji Inoue from National Hospital Organization Kochi Hospital, Kochi; Soichi Itaba from Kyushu Rosai Hospital, Kitakyushu; Yasuyuki Kihara from Kitakyushu General Hospital, Kitakyushu; Koji Mori from Mori Clinic, Fukuoka; Shinichiro Yada from ONGA NAKAMA Medical Association ONGA Hospital, Onga; Yojiro Sadamoto from Sadamoto Clinic, Kitakyushu; Ryuichi Iwakiri from Saga Medical School, Saga; Takahiro Noda from Karatsu Red Cross Hospital, Karatsu; Seiji Tsunada from NHO Ureshino Medical Center, Ureshino; Ken Tanigawa from Tanigawa Clinic, Nagasaki; Yasuhiko Gotoh from Shinbeppu Hospital, Beppu; Hisanori Abe from Arita GI Hospital, Oita; Yasuo Hanamure from Hanamure Hospital, Ichikikushikino.

\section{Compliance with ethical standards}

Conflict of interest Yoshikazu Kinoshita received honoraria from Eisai Co., Ltd., Daiichi Sankyo Co., Ltd., Takeda Pharmaceutical Co., Ltd., Otsuka Pharmaceutical Co., Ltd. and AstraZeneca K.K., and received research grants from Eisai Co., Ltd., Daiichi Sankyo Co., Ltd., Takeda Pharmaceutical Co., Ltd., Otsuka Pharmaceutical Co., Ltd. and AstraZeneca K.K. Mototsugu Kato received honoraria from AstraZeneca K.K., Eisai Co., Ltd., Daiichi Sankyo Co., Ltd., Takeda Pharmaceutical Co., Ltd. and Otsuka Pharmaceutical Co., Ltd., and research grants from Abbott Japan Co., Ltd., Eisai Co., Ltd., Daiichi Sankyo Co., Ltd. and Astellas Pharma Co., Ltd. Mitsuhiro Fujishiro received honoraria from HOYA Co., Ltd., Takeda Pharmaceutical Co., Ltd., AstraZeneka K.K., Zeria Pharmaceutical Co., Ltd. and Daiichi Sankyo Co., Ltd., and received research grants from Takeda Pharmaceutical Co., Ltd. and EA Pharma Co., Ltd. Hironori Masuyama received research grants from Eisai Co., Ltd. and Takeda Pharmaceutical Co., Ltd. Yoshiumi Okubo is an employee of EA Pharma Co., Ltd. Seiichiro Hojo is an employee of Eisai Co., Ltd. Motoyasu Kusano received honoraria and research grants from Eisai Co., Ltd., EA Pharma Co., Ltd., Daiichi Sankyo Co., Ltd., Takeda Pharmaceutical Co., Ltd., AstraZeneca K.K., Otsuka Pharmaceutical
Co., Ltd., TSUMURA \& CO., Zeria Pharmaceutical Co., Ltd. and Astellas Pharma Co., Ltd. The following authors declare that they have no conflict of interest: Ryo Nakata, Hisanori Abe, Shinji Kumagai, and Yasushi Fukushima.

Funding This study was funded by Eisai, Co., Ltd., Tokyo, Japan and EA Pharma Co., Ltd., Tokyo, Japan. The writing and preparation of this paper were funded in part by EA Pharma. Writing support was provided by Eisai. Data analyses were undertaken by Eisai.

Open Access This article is distributed under the terms of the Creative Commons Attribution 4.0 International License (http://crea tivecommons.org/licenses/by/4.0/), which permits unrestricted use, distribution, and reproduction in any medium, provided you give appropriate credit to the original author(s) and the source, provide a link to the Creative Commons license, and indicate if changes were made.

\section{References}

1. Moki F, Kusano M, Mizuide M, et al. Association between reflux oesophagitis and features of the metabolic syndrome in Japan. Aliment Pharmacol Ther. 2007;26:1069-75.

2. Nakajima S, Nishiyama Y, Yamaoka M, et al. Changes in the prevalence of Helicobacter pylori infection and gastrointestinal diseases in the past 17 years. J Gastroenterol Hepatol. 2010;25(Suppl. 1):S99-110.

3. Kinoshita Y, Kawanami C, Kishi K, et al. Helicobacter pylori independent chronological change in gastric acid secretion in the Japanese. Gut. 1997;41:452-8.

4. Yagi S, Okada H, Takenaka R, et al. Influence of Helicobacter pylori eradication on reflux esophagitis in Japanese patients. Dis Esophagus. 2009;22:361-7.

5. Armstrong D. Systematic review: persistence and severity in gastro-oesophageal reflux disease. Aliment Pharmacol Ther. 2008;28:841-53.

6. Fujimoto K, Hongo M, Maintenance Study Group. Safety and efficacy of long-term maintenance therapy with oral dose of rabeprazole $10 \mathrm{mg}$ once daily in Japanese patients with reflux esophagitis. Intern Med. 2011;50:179-88.

7. Caro JJ, Salas M, Ward A. Healing and relapse rates in gastroesophageal reflux disease treated with the newer proton-pump inhibitors lansoprazole, rabeprazole, and pantoprazole compared with omeprazole, ranitidine, and placebo: evidence from randomized clinical trials. Clin Ther. 2001;23:998-1017.

8. Caos A, Breiter J, Perdomo C, et al. Long-term prevention of erosive or ulcerative gastro-oesophageal reflux disease relapse with rabeprazole 10 or $20 \mathrm{mg}$ vs. placebo: results of a 5-year study in the United States. Aliment Pharmacol Ther. 2005;22:193-202.

9. Fass R, Shapiro M, Dekel R, et al. Systematic review: protonpump inhibitor failure in gastro-oesophageal reflux diseasewhere next? Aliment Pharmacol Ther. 2005;22:79-94.

10. van der Velden AW, de Wit NJ, Quartero AO, et al. Maintenance treatment for GERD: residual symptoms are associated with psychological distress. Digestion. 2008;77:207-13.

11. Toghanian S, Johnson DA, Stålhammar NO, et al. Burden of gastro-oesophageal reflux disease in patients with persistent and intense symptoms despite proton pump inhibitor therapy: a post hoc analysis of the 2007 national health and wellness survey. Clin Drug Investig. 2011;31:703-15.

12. Peghini PL, Katz PO, Bracy NA, et al. Nocturnal recovery of gastric acid secretion with twice-daily dosing of proton pump inhibitors. Am J Gastroenterol. 1998;93:763-7. 
13. Kawamura M, Ohara S, Koike T, et al. The effects of lansoprazole on erosive reflux oesophagitis are influenced by CYP2C19 polymorphism. Aliment Pharmacol Ther. 2003;17:965-73.

14. Fujiwara Y, Kohata Y, Kaji M, et al. Sleep dysfunction in Japanese patients with gastroesophageal reflux disease: prevalence, risk factors, and efficacy of rabeprazole. Digestion. 2010;81:135-41.

15. Kusano M, Kouzu T, Kawano T, et al. Nationwide epidemiological study on gastroesophageal reflux disease and sleep disorders in the Japanese population. J Gastroenterol. 2008;43:833-41.

16. Kinoshita Y, Hongo M, Japan TWICE Study Group. Efficacy of twice-daily rabeprazole for reflux esophagitis patients refractory to standard once-daily administration of PPI: the Japan-based TWICE study. Am J Gastroenterol. 2012;107:522-30.

17. Horn J. Review article: relationship between the metabolism and efficacy of proton pump inhibitors-focus on rabeprazole. Aliment Pharmacol Ther. 2004;20(Suppl. 6):11-9.

18. Collen MJ, Johnson DA, Sheridan MJ. Basal acid output and gastric acid hypersecretion in gastroesophageal reflux disease. Correlation with ranitidine therapy. Dig Dis Sci. 1994;39:410-7.

19. Collen MJ, Strong RM. Comparison of omeprazole and ranitidine in treatment of refractory gastroesophageal reflux disease in patients with gastric acid hypersecretion. Dig Dis Sci. 1992;37:897-903.

20. Iwakiri K, Kinoshita Y, Habu Y, et al. Evidence-based clinical practice guidelines for gastroesophageal reflux disease 2015 . J Gastroenterol. 2016;51:751-67.

21. Armstrong D, Bennett JR, Blum AL, et al. The endoscopic assessment of esophagitis: a progress report on observer agreement. Gastroenterology. 1996;111:85-92.

22. Hongo M. Minimal changes in reflux esophagitis: red ones and white ones. J Gastroenterol. 2006;41:95-9.

23. Makuuchi H. Clinical study of sliding esophageal hernia-with special reference to the diagnostic criteria and classification of the severity of the disease. Nihon Shokakibyo Gakkai Zasshi. 1982;79:1557-67 (in Japanese, with English abstract).

24. Robinson M, Fitzgerald S, Hegedus R, FAST Trial Investigators, et al. Onset of symptom relief with rabeprazole: a community- based, open-label assessment of patients with erosive oesophagitis. Aliment Pharmacol Ther. 2002;16:445-54.

25. Jones MP. Acid suppression in gastro-oesophageal reflux disease: why? How? How much and when? Postgrad Med J. 2002;78:465-8.

26. Mody R, Bolge SC, Kannan H, et al. Effects of gastroesophageal reflux disease on sleep and outcomes. Clin Gastroenterol Hepatol. 2009;7:953-9.

27. Katelaris PH. An evaluation of current GERD therapy: a summary and comparison of effectiveness, adverse effects and costs of drugs, surgery and endoscopic therapy. Best Pract Res Clin Gastroenterol. 2004;18(Suppl):39-45.

28. Vakil N, van Zanten SV, Kahrilas P, Global Consensus Group, et al. The Montreal definition and classification of gastroesophageal reflux disease: a global evidence-based consensus. Am J Gastroenterol. 2006;101:1900-20.

29. Shimatani T, Inoue M, Kuroiwa $T$, et al. Rabeprazole $10 \mathrm{mg}$ twice daily is superior to $20 \mathrm{mg}$ once daily for night-time gastric acid suppression. Aliment Pharmacol Ther. 2004;19:113-22.

30. Kusano M, Sugimoto S, Kawamura O, et al. Numerical modification of the Los Angeles classification of gastroesophageal reflux disease fails to decrease observer variation. Dig Endosc. 2004;16:9-11.

31. Furuta K, Adachi K, Aimi M, et al. Effect of timing of proton pump inhibitor administration on acid suppression. Digestion. 2016;93:111-20.

32. Lee SW, Lee TY, Lien HC, et al. Comparison of risk factors and disease severity between old and young patients with gastroesophageal reflux disease. Gastroenterol Res. 2013;6:91-4.

33. Ashida K, Sakurai Y, Hori T, et al. Randomised clinical trial: vonoprazan, a novel potassium-competitive acid blocker, vs. lansoprazole for the healing of erosive oesophagitis. Aliment Pharmacol Ther. 2016;43:240-51.

34. Hongo M, Fujimoto K, Gastric Polyps Study Group. Incidence and risk factor of fundic gland polyp and hyperplastic polyp in long-term proton pump inhibitor therapy: a prospective study in Japan. J Gastroenterol. 2010;45:618-24. 